\title{
NOTE ON THE KLOOSTERMAN SUM
}

\author{
KENNETH S. WILLIAMS
}

Abstract. The Kloosterman sum

$$
\sum_{x=0 ;(x, p)-1}^{p^{\alpha}-1} \exp \left(2 \pi i n(x+\bar{x}) / p^{\alpha}\right)
$$

where $p$ is an odd prime, $\alpha \geqq 2$ and $(n, p)=1$, is evaluated in a very short direct way.

Let $p$ denote an odd prime, $\alpha$ a positive integer, and $n$ an integer such that $(n, p)=1$. The Kloosterman $\operatorname{sum} A_{p^{\alpha}}(n)$ is given by

$$
A_{p^{\alpha}}(n)=\sum_{x=0}^{p \alpha-1} \exp \left(2 \pi i n(x+\bar{x}) / p^{\alpha}\right),
$$

where the dash $\left({ }^{\prime}\right)$ indicates that $x$ only takes values from $0,1, \cdots$, $p^{\alpha}-1$ which are coprime with $p$, and $\bar{x}$ is the unique solution of the congruence $x \bar{x} \equiv 1\left(\bmod p^{\alpha}\right)$ satisfying $0<\bar{x}<p^{\alpha}$. Salié [3] has evaluated $A_{p^{a}}(n)$ explicitly when $\alpha \geqq 2$. His evaluation is based upon induction. A direct (but fairly long) proof has been given by Whiteman [4] which requires results concerning Ramanujan sums. In this note we give a modification of Salié's original argument which gives a very short direct evaluation of $A_{p^{a}}(n)$. (The referee has kindly pointed out that essentially the same technique has been used by Estermann [2], Carlitz [1].)

We let $\gamma=\alpha-[\alpha / 2]$ and $\delta=[\alpha / 2]$, so that $\alpha=\gamma+\delta, 2 \gamma \geqq \alpha$ and $\gamma$ $\geqq \delta \geqq 1$. Setting $x=u+v p^{\gamma}\left(u=0,1, \cdots, p^{\gamma}-1 ; v=0,1, \cdots, p^{\delta}-1\right)$ in (1), so that $\bar{x} \equiv \bar{u}-\bar{u}^{2} v p^{\gamma}\left(\bmod p^{\alpha}\right)$, we obtain

$$
\begin{aligned}
A_{p^{\alpha}}(n) & =\sum_{u=0}^{p^{\gamma}-1} \sum_{v=0}^{p^{\delta}-1} \exp \left(2 \pi i n\left(\left(u+v p^{\gamma}\right)+\left(\bar{u}-\bar{u}^{2} v p^{\gamma}\right)\right) / p^{\alpha}\right) \\
& =\sum_{u=0}^{p_{-1}^{\gamma}} \exp \left(2 \pi i n(u+\bar{u}) / p^{\alpha}\right) \sum_{v=0}^{p^{\delta}-1} \exp \left(2 \pi i n v\left(1-\bar{u}^{2}\right) / p^{\delta}\right) \\
& =p^{\delta} \sum_{u=0 ; u^{2} \equiv 1\left(\bmod p^{\delta}\right)}^{p^{\gamma-1}} \exp \left(2 \pi i n(u+\bar{u}) / p^{\alpha}\right)
\end{aligned}
$$

If $\alpha$ is even, say $\alpha=2 \beta$, then $\gamma=\delta=\beta$, and as the solutions $u$ of $u^{2} \equiv 1\left(\bmod p^{\beta}\right)$ in the range $0 \leqq u \leqq p^{\beta}-1$ are $u=1, p^{\beta}-1$ (so that

Received by the editors November 24, 1970.

AMS 1970 subject classifications. Primary 10G05. 
$\bar{u}=1, p^{2 \beta}-p^{\beta}-1$ respectively) we have

$$
A_{p} 2 \beta(n)=p^{\beta}\left\{\exp \left(4 \pi i n / p^{2 \beta}\right)+\exp \left(-4 \pi i n / p^{2 \beta}\right)\right\}=2 p^{\beta} \cos \left(4 \pi n / p^{2 \beta}\right) .
$$

If $\alpha$ is odd, say $\alpha=2 \beta+1$, then $\gamma=\beta+1, \delta=\beta$, and as the solutions $u$ of $u^{2} \equiv 1\left(\bmod p^{\beta}\right)$ in the range $0 \leqq u \leqq p^{\beta+1}-1$ are $u=1+w p^{\beta}$ $(w=0,1, \cdots, p-1),-1+w p^{\beta}(w=1,2, \cdots, p)$ (so that $\bar{u} \equiv 1$ $-w p^{\beta}+w^{2} p^{2 \beta},-1-w p^{\beta}-w^{2} p^{2 \beta}\left(\bmod p^{2 \beta+1}\right)$ respectively) we have

$$
\begin{aligned}
& A_{p^{2 \beta}+1}(n)=p^{\beta}\left\{\exp \left(4 \pi i n / p^{2 \beta+1}\right) \sum_{w=0}^{p-1} \exp \left(2 \pi i n w^{2} / p\right)\right. \\
& \left.+\exp \left(-4 \pi i n / p^{2 \beta+1}\right) \sum_{w=1}^{p} \exp \left(-2 \pi i n w^{2} / p\right)\right\} \\
& =2(n / p) p^{\beta+1 / 2} \cos \left(4 \pi n / p^{2 \beta+1}\right), \quad \text { if } p \equiv 1(\bmod 4) \text {, } \\
& =-2(n / p) p^{\beta+1 / 2} \sin \left(4 \pi n / p^{2 \beta+1}\right) \text {, if } p \equiv 3(\bmod 4) \text {, }
\end{aligned}
$$

using the well-known result [4]

$$
\sum_{w=0}^{p-1} \exp \left(2 \pi i n w^{2} / p\right)=(n / p) i^{(p-1)^{2} / 4} p^{1 / 2}
$$

\section{REFERENCES}

1. L. Carlitz, A note on multiple Kloosterman sums, J. Indian Math. Soc. 29 (1965), 197-200. MR 34 \#2532.

2. T. Estermann, On Kloosterman's sum, Mathematika 8 (1961), 83-86. MR 23 \#A3716.

3. H. Salié, Über die Kloostermanschen Summen $S(u, v ; q)$, Math. Z. 34 (1931), 91109.

4. A. L. Whiteman, A note on Kloosterman sums, Bull. Amer. Math. Soc. 51 (1945), 373-377. MR 6, 259.

Carleton University, Ottawa, Ontario, Canada 